\title{
Current Drive Calculations with an Advanced Adjoint Approach
}

\author{
N.B. Marushchenko, C.D. Beidler and H. Maassberg \\ Max-Planck-Institut für Plasmaphysik, EURATOM Association, \\ Teilinstitut Greifswald, D-17491 Greifswald, Germany
}

Contact person: Nikolai B. Marushchenko

Wendelsteinstrasse 1, D-17491 Greifswald, Germany

Tel: +49-3834-882330

Fax: +49-3834-882509

e-mail: nikolai.marushchenko@ipp.mpg.de

Number of pages: 15

Number of figures: 3 


\title{
Current Drive Calculations with an Advanced Adjoint Approach
}

\author{
N.B. Marushchenko*, C.D. Beidler and H. Maassberg \\ Max-Planck-Institut für Plasmaphysik, EURATOM Association, \\ Teilinstitut Greifswald, D-17491 Greifswald, Germany
}

\begin{abstract}
The advanced adjoint approach for arbitrary collisionalities with momentum conservation in the likeparticle collision is considered. The results are generally applicable for the parallel conductivity as well as for current-drive calculations. In addition, the weakly relativistic extension of the variational principle for the Spitzer function with momentum conservation in the electron-electron collision is described. The models developed are well suited to ray-tracing calculations.
\end{abstract}

*e-mail nikolai.marushchenko@ipp.mpg.de 


\section{INTRODUCTION}

The adjoint approach is commonly used for calculations of the current drive (CD) efficiency in ray-tracing [1-3] as well as for the momentum correction of the parallel conductivity and bootstrap current $[4,5]$. The key point is the choice of the model for the corresponding Spitzer function. The classical Spitzer problem in the collisional limit [6] with $\nu_{e}^{*} \gg 1$ can be analytically generalized to the collisionless limit [7] with $\nu_{e}^{*} \ll 1$ (here $\nu_{e}^{*}=\nu_{e} R / t v$ is the collisionality, $\nu_{e}$ is the collision frequency, $R$ and $t$ are the major radius and the rotational transform, respectively). The first limit, without trapped particle effects, i.e. $\nu_{e} \gg \tau_{b}^{-1}\left(\tau_{b}\right.$ is the electron bounce-time), gives the upper limit for CD efficiency, while the second one (collisionless, i.e. $\nu_{e} \ll \tau_{b}^{-1}$ ) tends to underestimate it. The intermediate collisional regime requires special attention, in particular for stellarators.

Calculations of even mono-energetic transport coefficients are rather computationally expensive in the collisionless limit. The treatment of the linearized collision operator with momentum conservation in like-particle collisions would require the solution of the drift-kinetic equation (DKE) in 4D-phase space instead of the 3D mono-energetic solution. Consequently, momentum correction techniques (e.g. in Refs. 4, 5) based on mono-energetic transport coefficients become attractive. In the 3D DKE, the flux surface label and the velocity are only parameters and the simple Lorentz pitch-angle collision term (without momentum conservation) is used, e.g. in the DKES code [8]. To include collisionality effects in the Spitzer function, the solution of the 3D DKE (2D for axisymmetric tokamaks) is mandatory. It is important to note, that the CD is much more localized in momentum space than the (integral) parallel conductivity, and hence much more sensitive to the model employed.

For ECRH/ECCD scenarios with high electron temperature, electrons interacting with the 
RF-field are largely in the lmfp-regime. For ECCD predictions, the "high-speed-limit" (see, e.g. Ref. 3), below abbreviated as $h s l$-model, is typically applied, where the Spitzer function is calculated for $v / v_{\mathrm{th}} \gg 1$ (here, $v_{\mathrm{th}}=\sqrt{2 T_{e} / m_{e}}$ ). Since the influence of supra-thermal electrons on momentum conservation is negligible, the DKE can be significantly simplified (in particular, the integral part of the collision operator is omitted in this approach).

The Spitzer function with parallel momentum conservation in the like-particle collisions ( $m c$-model) is the direct solution of the Fokker-Planck equation. It is also convenient to obtain a numerical fit derived from the variational principle based on the collisional entropy production with momentum conservation in the electron-electron collision term $[9,10]$, which approximates the solution of the corresponding Spitzer problem. This approximation is sufficiently accurate for the range $0.5 \lesssim v / v_{\text {th }} \lesssim 4$, i.e. for electrons responsible for the main cyclotron absorption. So far, this model has been developed only for the non-relativistic case. In the present work, the model of Ref. 10 is extended to the weakly relativistic case.

\section{DESCRIPTION OF THE MODEL}

Within the adjoint approach [1-3], the electron current, $j_{\|}=-e n_{e} \int \mathrm{d} \mathbf{u} v_{\|} f_{e}$, driven by the RF-field, can be expressed as $j_{\|}=e n_{e} v_{\mathrm{th}} /\left(\nu_{e 0} b\right) \cdot \int \mathrm{d} \mathbf{u}\left(g / F_{e M}\right) Q_{\mathrm{RF}}\left(F_{e M}\right)$, where $u=v \gamma / v_{\mathrm{th}}$ with $\gamma$ the Lorentz factor, $F_{e M}(u)=(\pi / 2 \mu)^{1 / 2} / K_{2}(\mu) \cdot \mathrm{e}^{-\mu \gamma}$ is the relativistic Maxwellian with $\mu=m_{e} c^{2} / T_{e}$ and $K_{2}$ the modified Bessel function of the second kind, $\nu_{e 0}=4 \pi n_{e} e^{4} \ln \Lambda /\left(m_{e}^{2} v_{\mathrm{th}}^{3}\right)$ the collision frequency, and $b=B / B_{\max }$ the normalized magnetic field, where $B_{\max }$ is the maximal value of $B$ on the given magnetic surface. The adjoint kinetic equation for the Green's function $g$ can be formulated as

$$
v_{\|} \mathbf{b} \cdot \nabla g+C^{l i n}(g)=\nu_{e 0} \frac{v_{\|}}{v_{\text {th }}} b F_{e M}
$$


with $C^{l i n}(g)=C^{e e}\left(g ; F_{e M}\right)+C^{e e}\left(F_{e M} ; g\right)+C^{e i}\left(g ; F_{i M}\right)$ the linearized collision operator. Since only the 1st Legendre harmonic of $g$ is necessary for current drive calculation, the collision operator $C^{\text {lin }}$ is approximated by neglecting all the $n>1$ terms of the general Legendre series. To be rigorous, the last assumption is exactly correct only for straight magnetic field lines (or in the collisional limit), while for a toroidal plasma the equation for the 1st Legendre harmonic is coupled with higher harmonics [11]. In practice, nevertheless, in the collisionless limit, accuracy of this approach with only the 1st Legendre harmonic is about $0.05 \sqrt{\epsilon}[11]$ for tokamaks with $\epsilon$ the inverse aspect ratio, which is sufficient accuracy for the problem considered. The same can be argued for the stellarators, since only the global maximum and minimum of $B$ define the fraction circulating particles, and, consequently, the problem considered for stellarators with low collisionality is qualitatively very similar to the tokamaks case.

Considering at the moment only the collisionless limit, the solution of Eq. (1) for the 1 st Legendre harmonic can be represented as $[3,7,10]$

$$
\begin{aligned}
& g_{1}(u, \lambda)=-\operatorname{sign}\left(u_{\|}\right) H(\lambda) K(u), \\
& H(\lambda)=\frac{1}{2 f_{c}} \Theta(1-\lambda) \int_{\lambda}^{1} \frac{\mathrm{d} \lambda}{\langle\sqrt{1-\lambda b}\rangle},
\end{aligned}
$$

where $\lambda=u_{\perp}^{2} / u^{2} b$ is the normalized magnetic moment, $\Theta(y)=1$ for $y \geq 0$ (passing particles), while $\Theta(y)=0$ for $y<0$ (trapped particles), $f_{c}=\frac{3}{4}\left\langle b^{2}\right\rangle \int_{0}^{1} \frac{\lambda d \lambda}{\langle\sqrt{1-\lambda b}\rangle}$ is the fraction of circulating (passing) particles, $\langle\ldots\rangle$ denotes flux surface averaging, and $K(u)$ is the Spitzer function (in this paper, when it is appropriate, also the Spitzer function normalized to the Maxwellian, $\chi(u)=$ $K / F_{e M}$, is used). The equation for the Spitzer function can be formulated then as [7, 10]

$$
C_{1}^{e}(K)-\frac{f_{\operatorname{tr}}}{f_{c}}\left(\nu_{e e}(u)+\nu_{e i}(u)\right) K(u)=\nu_{e 0} \frac{u}{\gamma} F_{e M}
$$

where $C_{1}^{e}(K)$ is the 1 st Legendre harmonic of the linearized collision operator, and $f_{\operatorname{tr}}=1-f_{c}$ is the fraction of trapped particles. 
Formally, this model is applicable only for the collisionless and collisional limits (in the last case, $f_{\operatorname{tr}}=0$ and $H(\lambda)=\left|u_{\|}\right| / u$ ). Below, the model based on Eq. (3) is extended i) for arbitrary collisionalities, and ii) by modification of the variational principle [10] for the relativistic collision operator [12].

\section{A. Spitzer function for finite collisionalities}

Let us consider the collisionless Spitzer function, $K(x)$, defined by the 1D integrodifferential equation (3) in the non-relativistic case with $\gamma=1$ and $u=x \equiv v / v_{\text {th }}$. The DKES code [8] (Drift-Kinetic Equation Solver) calculates the 3 mono-energetic transport coefficients; of particular interest here is the dimensionless mono-energetic parallel electric conductivity, $\sigma_{\|}^{\mathrm{me}}(x)$. The collisional limit, which is equivalent to the straight magnetic field line case with $f_{c}=1$, corresponds to the limit $\sigma_{\|}^{\mathrm{me}} / \nu^{*}=1$ (here, $\nu^{*}=\nu(x) / x$ is the mono-energetic "collisionality"), while in the collisionless limit $\sigma_{\|}^{\mathrm{me}} / \nu^{*}$ is reduced to the passing (circulating) particle fraction, $f_{c}$, i.e. the trapped particles defined by $f_{\text {tr }}$ do not contribute to the parallel current. With electronelectron momentum conservation, a stronger weighting of the trapped particles defined by $f_{\mathrm{tr}} / f_{c}$ in Eq. (3) appears which reflects the passing-trapped electron friction adding to the friction with ions and impurities. In a very natural extension, $f_{c}$ in Eq. (3) is replaced by the "effective" passing particle fraction, $f_{c}^{\text {eff }}(x)=\sigma_{\|}^{\text {me }}(x) / \nu^{*}(x)$, from the DKES data thus allowing for a collisionality dependence. A similar procedure was used in the fit representation of the normalized $\sigma_{\|}$in Ref. 13 where a 3D DKE (for tokamaks) with the full linearized collision operator was solved. This approach describes correctly both the collisional and collisionless limits. The current diffusion into the trapped-particle domain is reflected by the ratio of the collision frequency to the bounce frequency and depends on the specific magnetic configuration. This feature is well described by the DKES solution for $\sigma_{\|}^{\mathrm{me}}(x)$. 
In Fig.1, solutions of Eq. (3) with $f_{c}^{\text {eff }}(x)$ obtained from the DKES data for the "standard" W7-X configuration are shown. Solutions are obtained for $Z_{\text {eff }}=1$ (left) and $Z_{\text {eff }}=2$ (right), with fixed density, $n_{e}=1 \times 10^{20} \mathrm{~m}^{-3}$, and for different electron temperatures, that corresponds to the different collisionalities (for collisional and collisionless limits, $T_{e}=0$ and $T_{e}=100 \mathrm{keV}$, respectively, are applied; please, note, that so high upper temperature is required just to cover the collisionless limit, while the relativistic effects are omitted). As expected, the resulting Spitzer functions lie in the range between the collisional and collisionless limits.

In order to benchmark the model, the (thermal) parallel electric conductivity is calculated for a tokamak case. Fig.2 (left) shows the normalized conductivities calculated from the solution of Eq. (3) with $f_{c}^{\text {eff }}(x)$ together with simple fit formulas for tokamaks with momentum conservation taken into account. The collisionless limit as well as the transitions from the plateau- to the PfirschSchlüter and the banana-regime are given for reference. The Hinton-Hazeltine approximation [14] (dot-dashed line) is valid only for large aspect ratios $\left(\epsilon_{t} \ll 1\right)$ whereas the agreement with Ref. 13 (dashed line) is rather good even for larger $\epsilon_{t}$. Consequently, this momentum correction technique based on the (mono-energetic) $f_{c}^{\text {eff }}(x)$ from DKES can be expected to handle complex stellarator configurations, as well.

Fig.2 (right) shows the comparison of $\sigma_{\|}\left(\nu_{e}^{*}\right)$ for the "standard" W7-X configuration and the equivalent axisymmetric configuration with the strong W7-X elongation taken into account. For W7-X, the conductivity is dominated by both the helical ( $B_{11}$-Fourier component) and the toroidal mirror term $\left(B_{01}\right.$ term); the toroidal curvature $\left(B_{10}\right.$ term) is rather small (high elongation). In general, the functional dependence of $\sigma_{\|}\left(\nu_{e}^{*}\right)$ for stellarators and tokamaks can be fairly different which reflects the bounce frequencies of the different types of trapped particles.

At first consideration, introducing the concept of an "effective" passing particle fraction as a function of energy into Eq. (3) is in some contradiction with the original formulation given in 
Eq. (2), where $f_{c}$ is strictly defined by the magnetic configuration alone. Nevertheless, despite the fact that, formally, trapped particles are excluded from consideration in Eq. (2), their contribution is included implicitly as an integral effect in the present Spitzer function, $K(u)$, since the DKES solution gives the mono-energetic parallel conductivity accounting for the (barely) trapped particle contribution. In this approach, the shape of the Spitzer function is definitely determinated by the total fraction of current driving particles dependent on energy.

\section{B. Weakly relativistic Spitzer function}

The Coulomb coefficients of the fully relativistic operator [12] have an additional parametric dependence on $\mu=m c^{2} / T_{e} \gg 1$. Since the exact fully relativistic Coulomb coefficients are rather complex for calculations, we simplify the analytical expressions by an expansion of the collision operator in a power series in $\mu^{-1}$,

$$
C_{1}^{e}=C_{1, \mu=\infty}^{e}+\mu^{-1} C_{1,1}^{e}+\mu^{-2} C_{1,2}^{e}+O\left(\mu^{-3}\right) .
$$

The main advantage of this representation is that the final formulas (see below) can be easily expressed through the standard integrals.

With this expansion, the upper electron energy limit is introduced, $u_{\max }^{2} / \mu \lesssim 1$ (for the ITER conditions with $\mu \simeq 20$ it gives $u_{\max } \lesssim 4.5$ ). Since the main cyclotron absorption happens usually in the range $u \sim 1-4$, this expansion is quite sufficient.

Following Refs. 9, 10, the solution of Eq. (3) is found from the variational principle by minimization of the collisional entropy production. Approximating the solution by the test function of Refs. 9, 10 modified by the factor $1 / \gamma$,

$$
\chi(u) \simeq \chi_{a}(u)=\frac{u}{\gamma} \sum_{i=1}^{4} d_{i} u^{i}
$$


the functional

$$
\begin{aligned}
S\left[\chi_{a}\right]=\int \mathrm{d}^{3} u & {\left[\frac{\chi_{a}}{F_{e M}} C_{1}^{e}\left(\chi_{a} F_{e M}\right)-\frac{f_{\mathrm{tr}}}{f_{c}} \frac{\chi_{a}}{F_{e M}} \nu_{e}(u) \chi_{a}-2 \nu_{e 0} \frac{u_{\|}}{\gamma} \chi_{a}\right.} \\
& \left.-2 \zeta\left(u_{\|} C_{1}^{e}\left(\chi_{a} F_{e M}\right)-\frac{f_{\mathrm{tr}}}{f_{c}} \nu_{e}(u) \chi_{a}-2 \nu_{e 0} \frac{u u_{\|}}{\gamma} F_{e M}\right)\right],
\end{aligned}
$$

is minimized with respect to $d_{i}$ leading to the system of $4+1$ equations for $d_{i}$ and $\zeta$,

$$
\begin{aligned}
\left(M_{i j}+\Omega_{i j}\right) d_{j}+\left(M_{0 i}+\Omega_{0 i}\right) \zeta & =G_{i}, \\
\left(M_{0 j}+\Omega_{0 j}\right) d_{j}+ & 0 \cdot \zeta=G_{0}
\end{aligned}
$$

(similar to Refs. 9, 10, the term proportional to $\zeta$ in Eqs. (6) and (7) is added to guarantee parallel momentum conservation). Here, $\nu_{e}(u) \equiv \nu_{e e}(u)+\nu_{e i}(u)$. It is important to mention here, that the test function Eq. (5) does not cover exactly the asymptotic for $u \gg 1$, especially for low temperatures, when $\gamma \simeq 1$ and $\chi_{a} \sim u^{5}$ instead of the high-speed-limit $\chi_{\mathrm{hsl}} \sim u^{4}$. Nevertheless, its accuracy is sufficient for the most interesting range, $u<4$.

The definitions for the matrix coefficients, $M_{i j}, \Omega_{i j}$ and $G_{i}$, are very similar to the nonrelativistic formulation given in Ref. 10, with only the relativistic Coulomb operator taken in the approximation of Eq. (4) instead of the non-relativistic one,

$$
\begin{aligned}
M_{i j} & =-2 \int \mathrm{d}^{3} u \frac{u^{i+1}}{\Gamma_{i}} p C_{1}^{e}\left(\frac{u^{j+1}}{\gamma} p F_{e M}\right), \\
\Omega_{i j} & =2 \int \mathrm{d}^{3} u \frac{u^{i+j+2}}{\gamma \Gamma_{i}} p^{2} F_{e M}(u) \nu_{e}(u) \frac{f_{\mathrm{tr}}}{f_{c}} \\
G_{i} & =2 \nu_{e 0} \int \mathrm{d}^{3} u \frac{u^{i+2}}{\gamma \Gamma_{i}} p^{2} F_{e M}(u),
\end{aligned}
$$

(here, $p \equiv \cos \theta$ is the pitch, $\Gamma_{i}=\gamma$ for $i \geq 1$, and $\Gamma_{0}=1$ ). Despite the fact that the final explicit formulas for these coefficients (apart of $\Omega_{i j}$ ) are rather lengthy, they can be directly integrated (with help, for example, the Mathematica package). Please, find, that this formulation is suitable for arbitrary collisionality, where the "effective" passing particle fraction, $f_{c}^{\text {eff }}(x)$, can be applied. In the collisionless limit with $f_{c}^{\text {eff }}(x)=f_{c}$, the integration for $\Omega_{i j}$ can also be performed analytically. Since the relativistic effects in collisional plasma response are important only for very high 
electron temperatures, we consider here only the collisionless limit (see Fig.1)The final formulas for the matrix coefficients $M_{i j}, \Omega_{i j}$ and $G_{i}$ in this limit are given in the Appendix.

In Fig.3, the weakly relativistic Spitzer function obtained with accuracy $O\left(1 / \mu^{3}\right)$ from the variational principle for $T_{e}=25 \mathrm{keV}$ (full lines) together with the exact non-relativistic solution (dots) for collisionless limits with $f_{\mathrm{tr}}=0.5$ are shown. Even for such a high temperature, the approximate solution coincides well with exact non-relativistic one for low energies (apart of not so important $u \ll 1$ range), $u<2$ (Fig.3, left), but for higher energies the discrepancy becomes significant. For comparison, also the fully relativistic high-speed-limit solution (Eq. (33) in Ref.3) is shown (dash-dots). As expected, the discrepancy of high-speed-limit from momentum conserving solution for the bulk is large, and may lead to a significant underestimation of CD. As was mentioned above, even for high obliqueness, the main contribution in $\mathrm{CD}$ is coming from the electrons with velocities satisfying $u<4$, and higher energies are not important due to their negligible contribution in absorption.

The presented formulation for the weakly relativistic approach can be easily extended to the finite collisionality case by introducing the mono-energetic "effective" fraction of passing particles, $f_{c}^{\text {eff }}(x)$. Since the corresponding functional $S\left[\chi_{a}\right]$ in this case is the same as Eq. (6), the approach for solving the problem is equivalent, leading only to numerical calculations of the matrix coefficients. Nevertheless, the relativistic effect becomes important mainly in the lmfp-regime, and the present approach is quite sufficient.

\section{CONCLUSIONS}

It has been shown that the very simple "high-speed-limit" approach is in general not qualified for the estimation of the ECCD efficiency. This approach is truly applicable only for scenarios with sufficiently large launch angles in an optically thick plasma, where bulk electrons are surely not 
involved in the cyclotron interaction. Exact numerical solutions of the Spitzer problem as well as rather good approximations derived from the variational principle based on the collisional entropy production exist with momentum conservation in the electron-electron collision term. These approaches are valid also at lower velocities. A generalized formulation based on the non-relativistic solution of the mono-energetic drift-kinetic equation allows for arbitrary collisionalities and recovers the analytical formulation both in the collisional and the collisionless limit. In addition, the weakly relativistic extension of the variational principle for the collisionless Spitzer problem has been obtained. The numerical fit by the variational principle approximates the solution quite accurately in the main range of interest, $u \lesssim 4$. The weakly relativistic model has been successfully implemented in the ray-tracing code TRAVIS $[15,16]$. 


\section{APPENDIX A: MATRIX COEFFICIENTS FROM EQ. (8)}

The linearized Coulomb operator for $g_{1}=p F_{M} \chi_{1}$ with $p \equiv \cos \theta$, needed for the matrix coefficients defined in Eqs. (8), can be represented as

$$
\frac{C_{1}^{e}\left(p F_{M} \chi_{1}\right)}{p F_{M}}=\frac{1}{u^{2}} \frac{\partial}{\partial u}\left(u^{2} D_{u u, 0}^{e e} \frac{\partial \chi_{1}}{\partial u}\right)+F_{u, 0}^{e e} \frac{\partial \chi_{1}}{\partial u}-\frac{2}{u^{2}}\left(D_{\theta \theta, 0}^{e e}+D_{\theta \theta, 0}^{e i}\right) \chi_{1}+I^{e e}\left(F_{M} \chi_{1}\right)
$$

where the diffusion and friction Coulomb coefficients, $D_{u u, 0}^{e e}, D_{\theta \theta, 0}^{e e}$ and $F_{u, 0}^{e e}$ (see Ref. 12) are taken in the weakly relativistic approach with accuracy $O\left(\mu^{-3}\right)$, while $D_{\theta \theta, 0}^{e i}$ is taken in the standard Lorentz limit $m_{i} \rightarrow \infty$. The last term in Eq. (A1), $I^{e e}\left(F_{M} \chi_{1}\right)$, is the integral term responsible for conservation of the parallel momentum in electron-electron collisions, which is estimated here with the same accuracy. The collision frequency, i.e. the pitch scattering rate, $\nu_{e}(u)=\frac{2}{u^{2}}\left(D_{\theta \theta, 0}^{e e}+D_{\theta \theta, 0}^{e i}\right)$, is taken in the same approach.

Since the analytical expressions for matrix coefficients defined in Eqs. (8) seem to be rather cumbersome, we give here only the final practical formulas with accuracy $O\left(1 / \mu^{2}\right)$ (despite of the fact, that the results shown in Fig. 3 were obtained with accuracy $O\left(1 / \mu^{3}\right)$, in practise, nevertheless, it is very sufficient to use only 1st relativistic correction). For convenience, the coefficients are represented as

$$
\begin{aligned}
& M_{i j}=M_{i j}^{(0)}+\frac{1}{\mu} M_{i j}^{(1)}+O\left(\frac{1}{\mu^{2}}\right), \\
& \Omega_{i j}=\Omega_{i j}^{(0)}+\frac{1}{\mu} \Omega_{i j}^{(1)}+O\left(\frac{1}{\mu^{2}}\right), \\
& G_{i}=G_{i}^{(0)}+\frac{1}{\mu} G_{i}^{(1)}+O\left(\frac{1}{\mu^{2}}\right) .
\end{aligned}
$$

The symmetry relations are taken into account, $M_{i j}=M_{j i}, \Omega_{i j}=\Omega_{j i}$, and for $i, j \geq 1, \Omega_{i+k, j+l}=$ 
$\Omega_{i+l, j+k}$. The complete expressions are collected below:

$$
\begin{array}{ll}
M_{01}^{(0)}=\frac{\sqrt{\pi}}{2} Z_{\mathrm{eff}} ; & M_{01}^{(1)}=-\frac{3}{2 \sqrt{\pi}}+\frac{3 \sqrt{\pi}}{4} Z_{\mathrm{eff}} ; \\
M_{02}^{(0)}=Z_{\mathrm{eff}} ; & M_{02}^{(1)}=-\frac{105}{64 \sqrt{2}}+\frac{25}{8} Z_{\mathrm{eff}} ; \\
M_{03}^{(0)}=\frac{3 \sqrt{\pi}}{4} Z_{\mathrm{eff}} ; & M_{03}^{(1)}=-\frac{3}{\sqrt{\pi}}+\frac{15 \sqrt{\pi}}{4} Z_{\mathrm{eff}} ; \\
M_{04}^{(0)}=2 Z_{\mathrm{eff}} ; & M_{04}^{(1)}=-\frac{945}{256 \sqrt{2}}+\frac{57}{4} Z_{\mathrm{eff}} ; \\
M_{11}^{(0)}=-\frac{104}{15}+\frac{151}{15 \sqrt{2}}+Z_{\mathrm{eff}} ; & M_{11}^{(1)}=\frac{2071}{105}-\frac{197861}{6720 \sqrt{2}}+\frac{9}{8} Z_{\mathrm{eff}} ; \\
M_{12}^{(0)}=\frac{4}{\sqrt{\pi}}-\sqrt{\pi}+\frac{3 \sqrt{\pi}}{4} Z_{\mathrm{eff}} ; & M_{12}^{(1)}=-\frac{5}{\sqrt{\pi}}+\sqrt{\pi}+\frac{15 \sqrt{\pi}}{8} Z_{\mathrm{eff}} ; \\
M_{13}^{(0)}=-\frac{102}{5}+\frac{607}{20 \sqrt{2}}+2 Z_{\mathrm{eff}} ; & M_{13}^{(1)}=-\frac{1437}{140}-\frac{131497}{8960 \sqrt{2}}+\frac{33}{4} Z_{\mathrm{eff}} ; \\
M_{14}^{(0)}=\frac{26}{\sqrt{\pi}}-7 \sqrt{\pi}+\frac{15 \sqrt{\pi}}{8} Z_{\mathrm{eff}} ; & M_{14}^{(1)}=\frac{52}{\sqrt{\pi}}-\frac{\sqrt{\pi}}{4}+\frac{45 \sqrt{\pi}}{4} Z_{\mathrm{eff}} ; \\
M_{22}^{(0)}=\sqrt{2}+2 Z_{\mathrm{eff}} ; & M_{22}^{(1)}=-\frac{1105}{256 \sqrt{2}}-\frac{15}{4} Z_{\mathrm{eff}} ; \\
M_{23}^{(0)}=\frac{6}{\sqrt{\pi}}+\frac{15 \sqrt{\pi}}{8} Z_{\mathrm{eff}} ; & M_{23}^{(1)}=-\frac{49}{2 \sqrt{\pi}}+\frac{69 \sqrt{\pi}}{8}-\frac{15 \sqrt{\pi}}{8} Z_{\mathrm{eff}} ; \\
M_{24}^{(0)}=\frac{11}{\sqrt{2}}+6 Z_{\mathrm{eff}} ; & M_{24}^{(1)}=-\frac{30245}{1024 \sqrt{2}}+\frac{3}{4} Z_{\mathrm{eff}} ; \\
M_{33}^{(0)}=-\frac{228}{5}+\frac{6147}{80 \sqrt{2}}+6 Z_{\mathrm{eff}} ; & M_{33}^{(1)}=\frac{1161}{14}-\frac{550017}{7168 \sqrt{2}}+\frac{3}{4} Z_{\mathrm{eff}} ; \\
M_{34}^{(0)}=\frac{45}{\sqrt{\pi}}-\frac{9 \sqrt{\pi}}{4}+\frac{105 \sqrt{\pi}}{16} Z_{\mathrm{eff}} ; & M_{34}^{(1)}=\frac{79}{4 \sqrt{\pi}}+\frac{255 \sqrt{\pi}}{4}+\frac{315 \sqrt{\pi}}{32} Z_{\mathrm{eff}} ; \\
M_{44}^{(0)}=\frac{157}{2 \sqrt{2}}+24 Z_{\mathrm{eff}} ; & M_{44}^{(1)}=-\frac{2754977}{4096 \sqrt{2}}+75 Z_{\mathrm{eff}} ; \\
\end{array}
$$

The final expressions for matrix coefficients $\Omega_{i j}$ are given here only for the collisionless 
limit, when $f_{\mathrm{tr}}$ is not a function of energy anymore, and, consequently, $\Omega_{i j}=\frac{f_{\mathrm{tr}}}{f_{c}} \omega_{i j}$, with

$$
\begin{array}{rlrl}
\omega_{01}^{(0)} & =\frac{1}{\sqrt{\pi}}+\frac{\sqrt{\pi}}{2} Z_{\mathrm{eff}} ; & \omega_{01}^{(1)} & =-\frac{9}{4 \sqrt{\pi}}+\frac{3 \sqrt{\pi}}{4}-\frac{3 \sqrt{\pi}}{4} Z_{\mathrm{eff}} ; \\
\omega_{02}^{(0)} & =\frac{1}{\sqrt{2}}+Z_{\mathrm{eff}} ; & \omega_{02}^{(1)} & =\frac{19}{16 \sqrt{2}}-\frac{7}{8} Z_{\mathrm{eff}} ; \\
\omega_{03}^{(0)} & =\frac{1}{\sqrt{\pi}}+\frac{\sqrt{\pi}}{4}+\frac{3 \sqrt{\pi}}{4} Z_{\mathrm{eff}} ; & \omega_{03}^{(1)}=\frac{7}{4 \sqrt{\pi}}+\frac{7 \sqrt{\pi}}{8} ; \\
\omega_{04}^{(0)}=\frac{9}{4 \sqrt{2}}+2 Z_{\mathrm{eff}} ; & \omega_{04}^{(1)}=\frac{591}{64 \sqrt{2}}+\frac{9}{4} Z_{\mathrm{eff}} ; \\
\omega_{11}^{(0)}=\omega_{02}^{(0)} ; & \omega_{11}^{(1)}=-\frac{17}{16 \sqrt{2}}-\frac{23}{8} Z_{\mathrm{eff}} ; \\
\omega_{12}^{(0)}=\omega_{03}^{(0)} ; & \omega_{12}^{(1)}=-\frac{3}{4 \sqrt{\pi}}+\frac{\sqrt{\pi}}{8}-\frac{15 \sqrt{\pi}}{8} Z_{\mathrm{eff}} ; \\
\omega_{13}^{(0)}=\omega_{04}^{(0)} ; & \omega_{13}^{(1)}=\frac{131}{64 \sqrt{2}}-\frac{15}{4} Z_{\mathrm{eff}} ; \\
\omega_{14}^{(0)}=\frac{5}{2 \sqrt{\pi}}+\frac{3 \sqrt{\pi}}{4}+\frac{15 \sqrt{\pi}}{8} Z_{\mathrm{eff}} ; & \omega_{14}^{(1)}=\frac{41}{8 \sqrt{\pi}}+\frac{15 \sqrt{\pi}}{8}-\frac{15 \sqrt{\pi}}{8} Z_{\mathrm{eff}} ; \\
\omega_{24}^{(0)}=\frac{115}{16 \sqrt{2}}+6 Z_{\mathrm{eff}} ; & \omega_{24}^{(1)}=\frac{7133}{256 \sqrt{2}}+\frac{3}{4} Z_{\mathrm{eff}} ; \\
\omega_{34}^{(0)}=\frac{9}{\sqrt{\pi}}+\frac{45 \sqrt{\pi}}{16}+\frac{105 \sqrt{\pi}}{16} Z_{\mathrm{eff}} ; & \omega_{34}^{(1)}=\frac{203}{4 \sqrt{\pi}}+\frac{525 \sqrt{\pi}}{32}+\frac{315 \sqrt{\pi}}{32} Z_{\mathrm{eff}} ; \\
\omega_{44}^{(0)}=\frac{1911}{64 \sqrt{2}}+24 Z_{\mathrm{eff}} ; & \omega_{44}^{(1)}=\frac{239853}{1024 \sqrt{2}}+75 Z_{\mathrm{eff}} .
\end{array}
$$

The final analytical expression for $G_{i}$ is very compact,

$$
G_{i}^{(0)}=\Gamma\left(\frac{5+i}{2}\right) ; \quad G_{i}^{(1)}=-\frac{15}{8} \Gamma\left(\frac{5+i}{2}\right)-2 \Gamma\left(\frac{7+i}{2}\right)+\frac{1}{2} \Gamma\left(\frac{9+i}{2}\right) .
$$

One can find, that the non-relativistic contributions (indexed above by zero) are the same as defined in $[9,10]$ (see Eq.21 in [9] for $M_{i j}^{(0)}$, and Eq.A21 and Eq.A22 in [10] for $\omega_{i j}^{(0)}$ and $G_{i j}^{(0)}$, respectively). 
[1] T.M. Antonsen and K.R. Chu, Phys. Fluids 25, 1295 (1982).

[2] T.M. Antonsen and B. Hui, IEEE Trans. Plasma Sci. PS-12, 118 (1984).

[3] Y.R. Lin-Liu, V.S. Chan, and R. Prater, Phys. Plasmas 10, 4064 (2003).

[4] M. Taguchi, Phys. Fluids B 4, 3638 (1992).

[5] H. Sugama and S. Nishimura, Phys. Plasmas 9, 4637 (2002).

[6] L. Spitzer and R. Härm, Phys. Rev. 89, 977 (1953).

[7] M. Taguchi, Plasma Phys. Control. Fusion 31, 241 (1989).

[8] W.I. van Rij and S.P. Hirshman, Phys. Fluids B 1, 563 (1989).

[9] S.P. Hirshman, Phys. Fluids 23, 1238 (1980).

[10] M. Romé et al, Plasma Phys. Control. Fusion 40, 511 (1998).

[11] C.F.F. Karney, N.J. Fisch, and A.H. Reiman, in AIP Conf. Proc. (1989), 190, p. 430.

[12] B.J. Braams and C.F.F. Karney, Phys. Fluids B 1, 1355 (1989).

[13] O. Sauter, C. Angioni, and Y.R. Lin-Liu, Phys. Plasmas 6, 2834 (1999).

[14] F.L. Hinton and R.D. Hazeltine, Rev. Mod. Physics 48, 239 (1976).

[15] N.B. Marushchenko et al, Plasma Fusion Res. 2, S1129 (2007), Spec. Issue: Proc. of the 16th Int. Toki Conf. on Advanced Imaging and Plasma Diagnostic, Toki, Japan, 5 - 8 December 2006.

[16] N.B. Marushchenko, H. Maassberg, and Y. Turkin, Nuclear Fusion 48, 054002 (2008). 
FIG. 1.

Spitzer functions, $\bar{K}(x)=\chi(x) \mathrm{e}^{-x^{2}}$, calculated for the "standard" W7-X configuration with $Z_{\text {eff }}=1$ (left) and $Z_{\text {eff }}=2$ (right), for different temperatures. Solid and dotted lines correspond to the collisionless limit with $T_{e}=100 \mathrm{keV}$ and collisional limit with $T_{e} \rightarrow 0$, respectively. Dashed, dot-dashed and dot-dot-dashed lines correspond to $1 \mathrm{keV}, 3 \mathrm{keV}$ and $10 \mathrm{keV}$.

FIG. 2 .

Left: parallel conductivity, $\sigma_{\|}$, normalized to the collisional Spitzer value vs the thermal electron collisionality for a tokamak with $\epsilon_{t}=0.05$ and $Z_{\text {eff }}=1$. Solid line: obtained from solution of Eq. (3) with $f_{c}^{\text {eff }}=\sigma_{\|}^{\text {me }} / \nu^{*}$ from DKES; dashed line: from Eq. (13) in Ref. 13; dash-dot line: from Eq. (6.122) in Ref. 14. Right: the same for the W7-X "standard" configuration (full line) and for the axisymmetric configuration (dot-dashed line) with identical toroidal curvature at half the plasma radius.

FIG. 3.

The Spitzer functions for $f_{\mathrm{tr}}=0.5$ and $Z_{\mathrm{eff}}=2$ are shown: weakly relativistic variational principle $m c$-solution (full line) and fully relativistic $h s l$-solution (dash-dots) for $T_{e}=25 \mathrm{keV}$, respectively. As reference, the exact non-relativistic solution (dots) is shown. Both Spitzer functions are shown, normalized by the Maxwellian, $\chi(u)$ (left), and $K(u)=\chi(u) F_{e M}$ (right). 

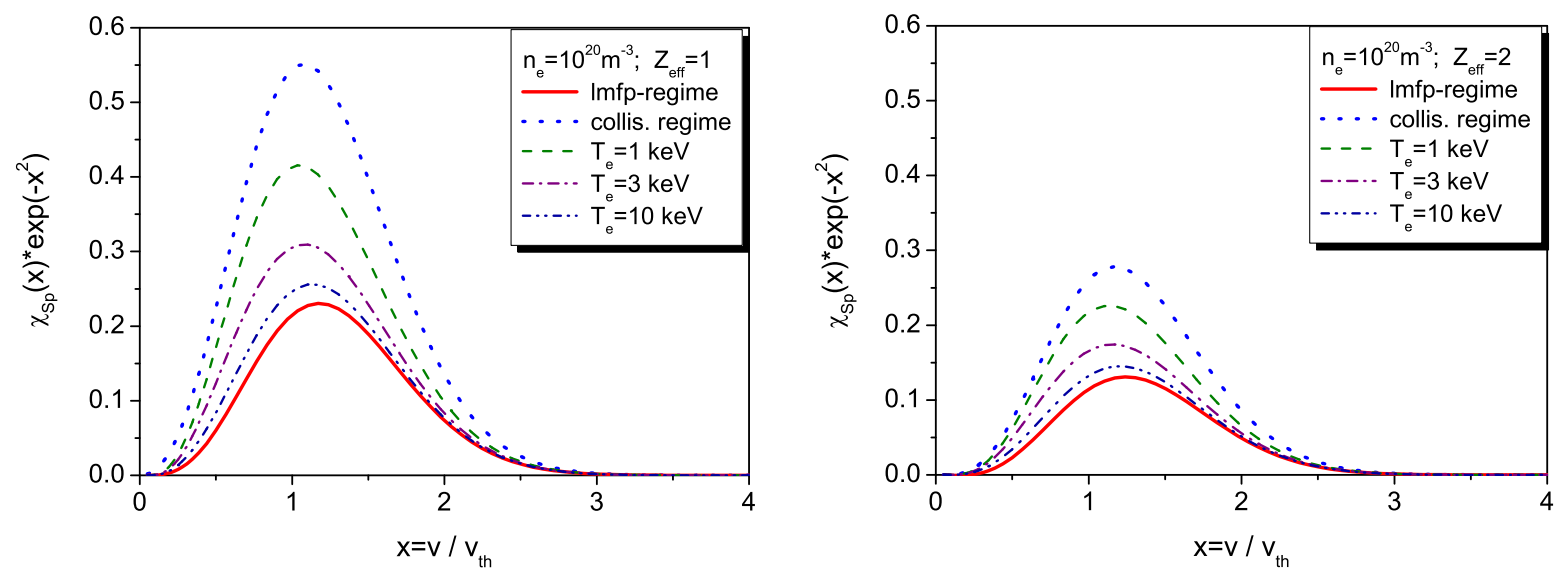

Fig.1 

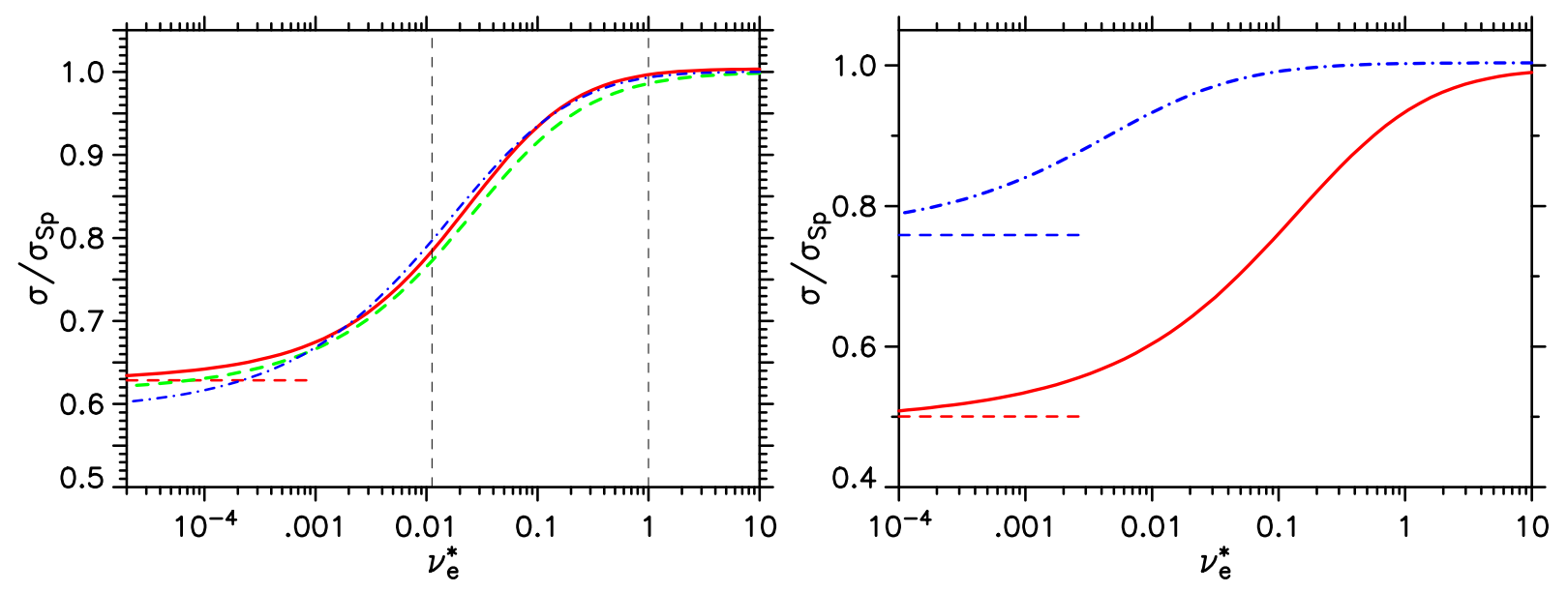

Fig.2 

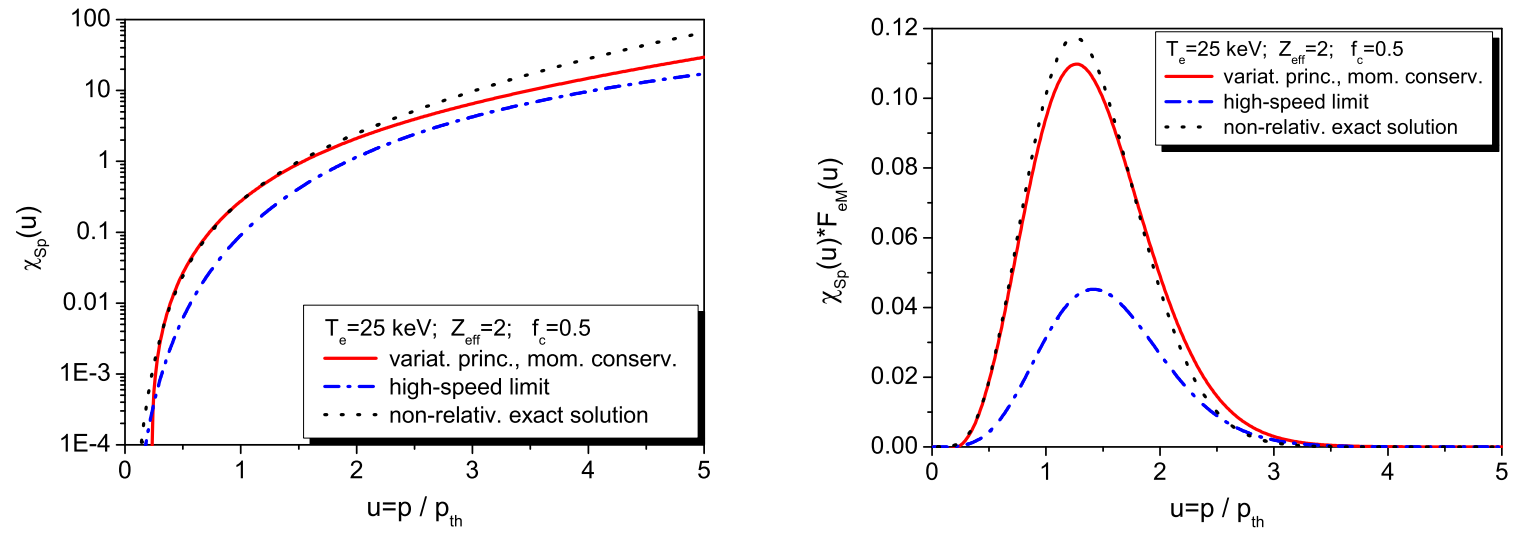

Fig.3 